\title{
LA UNIVERSIDAD: DIFUSIÓN Y PUBLICACIÓN CIENTÍFICA
}

\author{
THE UNIVERSITY: DISSEMINATION AND SCIENTIFIC PUBLICATION
}

Elio Iván Rodríguez-Chávez ${ }^{1, a}$

La Universidad Ricardo Palma, como Institución de educación superior líder, esta fuertemente comprometida con impulsar la difusión científica y la publicacion de revistas científicas de calidad. En la era del conocimiento, y del conocimiento instantáneo, contar con revistas científicas indizadas y disponibles online reporta enormes beneficios, tanto para la comunidad universitaria como para la comunidad científica en general.

La Revista de la Facultad de Medicina Humana de la Universidad Ricardo Palma presenta algunas particularidades importantes a señalar:

- Es una Revista que publica temas de Medicina y de Salud Publica.

- Pertenece a las Revistas Open Acces, que es adecuado para la realidad de Latinoamérica y pone al alcance de todos de manera libre, los últimos conocimientos y resultados de trabajos originales de investigación.

- Es un Revista Universitaria, relacionada a las ciencias medicas, una de las áreas de mayor producción a nivel internacional.

- Publica trabajos de calidad de diversos autores nacionales e internacionales.

- Alcanzó y cumplió diversos estándares nacionales e internacionales.

Por todo ello, en el contexto de los 50 Aniversarios de la Universidad Ricardo Palma, Felicitamos a todo el equipo editorial de la Revista de la Facultad de Medicina Humana, liderado por el Dr. Jhony A. De La Cruz Vargas, por las diversas indizaciones alcanzadas, las mas recientes en Scielo y LILACS. Fortalecer la visibilidad institucional y la internacionalización son objetivos claros de nuestra Universidad.

Quedan muchos desafíos en el futuro, mantenerse y aspirar a nuevas indizaciones son los próximos retos. La Universidad Ricardo Palma se congratula por estos logros que son inherentes a la visión y la misión de nuestra Universidad.

\section{Dr. Iván Rodríguez Chávez \\ Rector \\ Universidad Ricardo Palma}

\footnotetext{
${ }^{1}$ Universidad Ricardo Palma, Lima-Perú.

${ }^{\text {a Rector }}$

Citar como: Elio Iván Rodríguez-Chávez. La universidad: difusión y publicación científica. Rev. Fac. Med. Hum. Octubre 2019; 19(4):9 DOI 10.25176/ RFMH.v19i4.2333 